\title{
An Analytical Formula for the Mean Differential Group Delay of Randomly Birefringent Spun Fibers
}

\author{
Andrea Galtarossa, Member, IEEE, Luca Palmieri, Anna Pizzinat, Brian S. Marks, and \\ Curtis R. Menyuk, Fellow, IEEE, Fellow, OSA
}

\begin{abstract}
Polarization-mode dispersion (PMD) is a serious impairment for high-bit-rate optical telecommunication systems. It is known that spinning the fiber during the drawing process drastically reduces the PMD. However, the analysis of pulse propagation through a randomly birefringent spun fiber is still at an early stage. In this paper, we derive an analytical formula for the mean differential group delay of a periodically spun fiber with random birefringence. We model the birefringence with fixed modulus and a random orientation under the condition that the spin period is shorter than the beat length. Finally, we numerically compare the analytical results with those given by the random-modulus model of birefringence, and we obtain good agreement as long as the short-period assumption is satisfied.
\end{abstract}

Index Terms-Beat length, birefringence correlation length, fiber birefringence, polarization-mode dispersion (PMD), spun fibers.

\section{INTRODUCTION}

$\mathbf{T}$ HE INTEREST in polarization-mode dispersion (PMD) dates back more than 20 years [1], and, since then, this interest has grown as the capacity of optical telecommunication systems has increased. Indeed, PMD is currently considered to be one of the most serious impairments in high-bit-rate systems [2].

Various aspects of PMD have been studied. These aspects include theoretical studies of its origin, its theoretical and experimental characterization, experimental measurement techniques, evaluation of its detrimental effects, and its mitigation. In this paper, we focus on its mitigation [2].

There are two different basic approaches to mitigation. The first is to compensate the PMD in already installed systems [3], [4]. Because the PMD fluctuates in time, the compensation must be active, greatly complicating the development of compensators and adding substantially to their expense. Nonetheless, commercial compensators are now available for systems at

Manuscript received September 27, 2002; revised April 14, 2003. This work was supported in part by the European project IST/ATLAS and by the MIUR 40\% Project 2001098217-002.

A. Galtarossa and L. Palmieri are with the Department of Information Engineering, University of Padova, Padova 35131, Italy.

A. Pizzinat is with the Department of Information Engineering, University of Padova, Padova 35131, Italy, and also with the Department of Computer Science and Electrical Engineering, the University of Maryland of Baltimore County, Baltimore, MD 21250 USA.

B. S. Marks is with the Department of Computer Science and Electrical Engineering, University of Maryland of Baltimore County, Baltimore, MD 21250 USA, and also with the Laboratory of Physical Sciences, College Park, MD 20740 USA.

C. R. Menyuk is with the Department of Computer Science and Electrical Engineering, University of Maryland of Baltimore County, Baltimore, MD 21250 USA.

Digital Object Identifier 10.1109/JLT.2003.814385
$10 \mathrm{~Gb} / \mathrm{s}$ and are being developed for systems at $40 \mathrm{~Gb} / \mathrm{s}$. The second approach is to design low-PMD fibers, which may be done by spinning the fiber as it is drawn [5]. While it has been found empirically that it is more effective to spin fibers at a rate that changes periodically as they are drawn [6]-[8], rather than spinning them at a constant rate, the reasons for the advantage of periodic over constant spinning have not been clear. A great part of the analysis to date has treated spun fibers with a constant birefringence and no random variations [5]-[9]. This case is a useful starting point for the study of spun fibers because the analysis is simple; however, it does not correspond to the usual case in telecommunication fibers.

In a recent paper [10], we provided, without proof, a formula [(9) in this paper] for the calculation of the mean differential group delay (DGD) of a periodically spun fiber. In this paper, we report the complete demonstration of this formula, which is obtained assuming that the spin period is short compared with the fiber beat length and also that the strength of the birefringence is constant, while its orientation is random and the fiber correlation length for the random orientations can have any value.

In Section II, we introduce two different models for describing fiber birefringence. Then, in Section III, we calculate analytically and numerically the mean DGD of periodically spun fibers. In particular, we discuss the implications of (9) for the DGD, which holds in the short period limit, and we discuss the consequences of choosing one model of birefringence over the other. Finally, Section IV contains a derivation of (9).

\section{Two MODELS FOR THE BIREFRINGENCE}

The evolution along the fiber of the polarization dispersion vector $\Omega(z, \omega)$ is governed by the dynamical equation [11]

$$
\frac{\partial \boldsymbol{\Omega}(z, \omega)}{\partial z}=\frac{\partial \boldsymbol{\beta}(z, \omega)}{\partial \omega}+\boldsymbol{\beta}(z, \omega) \times \boldsymbol{\Omega}(z, \omega)
$$

where $\omega$ is the angular frequency and $\boldsymbol{\beta}(z, \omega)=\left(\beta_{1}, \beta_{2}, \beta_{3}\right)^{\top}$ is the local birefringence vector. In standard telecommunication fibers, $\boldsymbol{\beta}(z, \omega)$ is a random function of $z$, whose properties may be described by appropriate statistical models. In this paper, we consider the two models proposed in [12], which are described here briefly for completeness.

First of all, we remark that both models assume that no circular birefringence is present, i.e., $\boldsymbol{\beta}=\left(\beta_{1}, \beta_{2}, 0\right)^{\top}$. This assumption is not restrictive, because usually circular birefringence of telecommunication fibers is negligible [13]. Moreover, these models also assume that only the modulus of birefringence depends on $\omega$, so that we can write $\beta(z, \omega)=b(z, \omega) \hat{\beta}(z)$, where $b(z, \omega)$ is the modulus and $\hat{\beta}(z)$ the direction. From a 
theoretical standpoint, it is not necessary to further specify the dependence of the modulus of birefringence on the optical frequency. In fact, in the analysis that we carry out, we denote the $\omega$ derivative of $b(z, \omega)$ as $b_{\omega}$, without making any assumptions about it. Nevertheless, in our numerical simulations, we must specify the frequency evolution of the birefringence, and, as usual, we assume that

$$
b_{\omega}(z)=\frac{\partial b(z, \omega)}{\partial \omega}=\frac{b(z, \omega)}{\omega} .
$$

The first model that we consider is the random-modulus model (RMM), in which the two components of the birefringence $\beta_{1}(z)$ and $\beta_{2}(z)$ are independent Langevin processes [12]

$$
\frac{d \beta_{i}}{d z}=-\rho \beta_{i}(z)+\sigma \eta_{i}(z), \quad i=1,2
$$

where $\eta_{1}(z)$ and $\eta_{2}(z)$ are independent white-noise sources. As a consequence, the modulus $b(z, \omega)$ is a Rayleigh distributed random variable. Parameters $\rho$ and $\sigma$ define the statistical properties of the birefringence. In particular, the birefringence correlation length $L_{F}$ equals $1 / \rho$, and the beat length $L_{B}$ equals $2 \pi /\left\langle b^{2}\right\rangle^{1 / 2}=2 \pi \sqrt{\rho} / \sigma$. Furthermore, in the long-length regime, the mean DGD may be written [12]

$$
\langle\Delta \tau(z)\rangle=\sqrt{\frac{16 z L_{F}}{3 \pi}\left\langle b_{\omega}^{2}(z)\right\rangle}=\frac{8}{\omega L_{B}} \sqrt{\frac{\pi}{3} z L_{F}}
$$

where the last equality is obtained using (2).

The RMM is the only fiber model that has been proposed to date that is consistent with experimental results [14], [15]. However, its analysis is complex. For this reason, we consider also the simpler fixed-modulus model (FMM), which assumes that the local birefringence vector equals

$$
\boldsymbol{\beta}(z, \omega)=b(\omega)(\cos 2 \theta(z), \sin 2 \theta(z), 0)^{\top}
$$

where $\theta(z)$ is a Wiener process, i.e., it obeys the equation $d \theta / d z=\sigma \eta(z)$. In this expression, $\sigma$ is a constant parameter, and $\eta(z)$ is a white-noise source [12]. In this model, the birefringence correlation length and the beat length are $L_{F}=1 /\left(2 \sigma^{2}\right)$ and $L_{B}=2 \pi / b$, respectively, and in the long-length regime, the mean DGD equals [12]

$$
\langle\Delta \tau(z)\rangle=\sqrt{\frac{16 z L_{F}}{3 \pi} b_{\omega}^{2}}=\frac{8}{\omega L_{B}} \sqrt{\frac{\pi}{3} z L_{F}} .
$$

Upon comparing (6) with (4), we note that the final expressions for the mean DGD are the same [12].

The two models that we have introduced, describe the random evolution of the intrinsic birefringence of a fiber before it is spun. When a spin is applied, the local birefringence vector undergoes a rotation. If we denote as $A(z)$ the spin function, i.e., the amplitude of the rotation imposed on the fiber, then the new birefringence vector reads $\mathrm{R}_{3}[2 A(z)] \boldsymbol{\beta}(z)$, where

$$
\mathrm{R}_{3}(\phi)=\left(\begin{array}{ccc}
\cos \phi & -\sin \phi & 0 \\
\sin \phi & \cos \phi & 0 \\
0 & 0 & 1
\end{array}\right)
$$

represents a rotation of amplitude $\phi$ around the vector $(0,0,1)^{\top}$. Moreover, as we already stated, only a periodic spin $A(z)$ is considered in this paper, and hereafter, $p$ represents the spin period.

\section{MEAN DGD OF SPUn FibERS}

In this section, we introduce the expression for the mean DGD of a randomly birefringent periodically spun fiber. We obtain the result using the FMM model of birefringence with the short-period assumption $p^{2} \ll L_{B}^{2}$. We also compare the results obtained with the FMM to those obtained numerically with the RMM, both when the short-period assumption is satisfied and when it is not.

In order to analyze the spin effects on fiber DGD, it is convenient to define a spin-induced reduction factor (SIRF), as the ratio between the DGD of a spun fiber and the DGD the same fiber would have if it were not spun, as follows:

$$
\operatorname{SIRF}=\frac{\langle\Delta \tau(z)\rangle}{\left\langle\Delta \tau_{\mathrm{un}}(z)\right\rangle} .
$$

\section{A. Within the Short-Period Assumption}

Let us consider a randomly birefringent fiber with a local birefringence vector that is modeled using the FMM, introduced in (5). If that fiber is spun according to a periodic function $A(z)$ of period $p$, it may be shown (see Section IV) that in the long-length regime, the mean DGD of the spun fiber equals

$$
\langle\Delta \tau\rangle=\sqrt{\frac{\mathcal{C}^{2}+\mathcal{S}^{2}}{\mathcal{C}}}\left\langle\Delta \tau_{\text {un }}\right\rangle .
$$

In (9), $\left\langle\Delta \tau_{\text {un }}\right\rangle$ is the mean DGD that the same fiber would have if it were not spun, and $\mathcal{C}$ and $\mathcal{S}$ are defined as

$$
\begin{aligned}
\mathcal{C}= & \frac{\gamma}{p^{2}} \int_{0}^{p} \int_{0}^{p} \exp \left(-2 \sigma^{2} u\right) \\
& \cos [2 A(t)-2 A(t-u)] d t d u \\
\mathcal{S}= & \frac{\gamma}{p^{2}} \int_{0}^{p} \int_{0}^{p} \exp \left(-2 \sigma^{2} u\right) \\
& \times \sin [2 A(t)-2 A(t-u)] d t d u
\end{aligned}
$$

where $\gamma=2 \sigma^{2} p /\left[1-\exp \left(-2 \sigma^{2} p\right)\right]$ and $2 \sigma^{2}=1 / L_{F}$. We remark that the expressions given in (10) depend only on the ratio $p / L_{F}$. To prove this result, it suffices to normalize $t$ and $u$ with respect to $p$.

The derivation of (9) will be given in Section IV. Here, we focus on some important aspects and consequences.

In the case that we are considering, the SIRF equals

$$
\operatorname{SIRF}=\Phi=\sqrt{\frac{\mathcal{C}^{2}+\mathcal{S}^{2}}{\mathcal{C}}} .
$$

It can be noted that $\Phi$ depends on $A(z)$ and on the ratio between the spin period and the birefringence correlation length, while it is independent of $L_{B}$ because of the short-period assumption. To evaluate (11), we calculate numerically the integrals given in (10) [16]. Fig. 1 refers to a sinusoidal spin profile $A(z)=$ $A_{0} \sin (2 \pi z / p)$ with $p=4 \mathrm{~m}$. The solid curves represent the evolution of $\Phi$ calculated according from (11) as a function of the spin amplitude $A_{0}$. From the upper curve to the lower curve, they correspond to $L_{F}=0.1,0.3,0.8$, and $3 \mathrm{~m}$, respectively, while the lowest solid curve corresponds to $L_{F} \rightarrow+\infty$.

We may notice that $\Phi$ is always less than 1, implying that the spin reduces the DGD in all cases. We confirmed numerically 


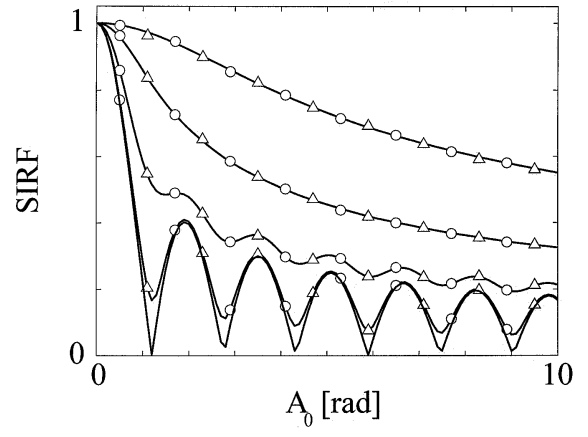

Fig. 1. Evolution of the SIRF as a function of spin amplitude, for a sinusoidal spin function with $p=4 \mathrm{~m}$ and $p^{2} \ll L_{B}^{2}$. The curves are obtained from (11). The solid curves, from the upper to the lower, correspond to $L_{F}=0.1,0.3,0.8$, and $3 \mathrm{~m}$, respectively. The lowest solid curve represents the case $L_{F} \rightarrow+\infty$. Triangles and circles are numerical estimates of the SIRF obtained from the FMM and the RMM, respectively, for $L_{B}=17.7 \mathrm{~m}$.

that this physically reasonable result holds for every spin function that we checked. Nevertheless, we have not been able to prove it analytically. From Fig. 1, we also see that the spin is less effective in reducing the DGD when $L_{F}$ is very short; in fact, in this case, the intrinsic random fluctuations of birefringence are very fast and tend to decrease the spin effect. For the same reason, we find that when $L_{F}$ is fixed and the spin amplitude increases, $\Phi$ tends to decrease.

As the birefringence correlation length increases, the variation of $\Phi$ versus $A_{0}$ presents well-defined minima and tends toward the case $L_{F} \rightarrow+\infty$. As was already observed for polarization-maintaining spun fibers [9], this result implies that if $L_{F}$ is known, it is much more effective to choose the spin rate properly, rather than spinning as fast as possible.

The expression for $\Phi$ in the case $L_{F} \rightarrow+\infty$ may be simplified. If $L_{F} \gg p$, then in both integrals (10), we may set $\exp \left(-2 \sigma^{2} u\right) \simeq 1$, and $\gamma \simeq 1$. As a consequence, one finds (see Appendix C) $\mathcal{S}=0$, and, therefore, the SIRF simplifies to $\Phi \simeq \sqrt{\mathcal{C}}$, where $\mathcal{C}$ can be rearranged as

$$
\mathcal{C} \simeq\left[\frac{1}{p} \int_{0}^{p} \cos 2 A(u) d u\right]^{2}+\left[\frac{1}{p} \int_{0}^{p} \sin 2 A(u) d u\right]^{2} .
$$

This specific case of polarization-maintaining fibers has been analyzed also by Chen et al. [17], using a different approach and yielding the same result. As we see from Fig. 1, when $L_{F} \rightarrow$ $+\infty$, there exist values of $A_{0}$ for which the reduction factor vanishes. It may be proved that these amplitudes correspond to those spins that make the DGD of a deterministic fiber a periodic function [18]. This result explains the numerical observation [9] that spin functions optimized for a polarization-maintaining fiber allow quasioptimal DGD reduction, even when the fiber birefringence evolves randomly. For example, we may calculate the mean DGD of a polarization-maintaining fiber spun according to the function $A(z)=A_{0} \sin (2 \pi z / p)$. According to what is stated above, we have $\langle\Delta \tau\rangle=\sqrt{\mathcal{C}}\left\langle\Delta \tau_{\text {un }}\right\rangle$, and using (12), we find $\mathcal{C}=J_{0}^{2}\left(2 A_{0}\right)$, where $J_{0}(x)$ is the zero-order Bessel function of the first kind. This result enables us to readily identify the optimal values of the spin amplitude.

It may be shown that there are two classes of spin functions for which (11) simplifies. The first of them is the class of spin

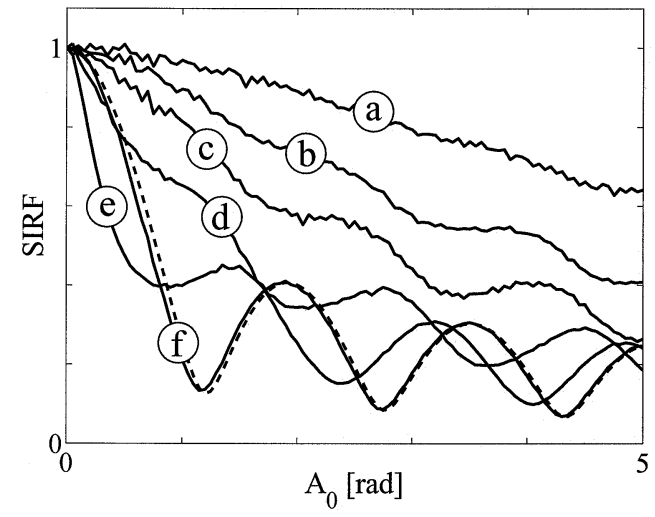

Fig. 2. Numerical estimates of the evolution of the SIRF with the RMM as a function of spin amplitude for a sinusoidal spin function with $p=L_{F}=4 \mathrm{~m}$. The solid curves, labeled with letters from a to $f$, correspond to $L_{B}=0.9$, $1.3,1.8,2.7,4.4$, and $17.7 \mathrm{~m}$, respectively. The dashed curve is obtained by calculating the SIRF using (11).

functions whose Fourier series contains only odd harmonics, for which one finds $A(z)=-A(z+p / 2)$ [9]. The second class consists of those functions that satisfy $A(z+q)=A(-z+q)$, where $q$ is an arbitrary quantity. If the spin function belongs to one of these two classes, then, exploiting the symmetry properties of $A(z)$, we show in Appendix $\mathrm{C}$ that $\mathcal{S}=0$. So that $\Phi=\sqrt{\mathcal{C}}$, notwithstanding that, in this case, $\mathcal{C}$ is still given by (10).

We tested the validity of (9) numerically and verified that it also holds for the more realistic RMM. To accomplish these tasks, we performed several numerical simulations for different spin functions and different values of birefringence parameters, and in all cases, we obtained a very good agreement for both the birefringence models. As an example, Fig. 1 shows numerical estimates of the mean DGD (circles and triangles) compared with (9) (solid curve). We implemented FMM and RMM and performed Monte Carlo simulations, solving (1) using the wave-plate model [19] over an ensemble of 6000 fibers $10 \mathrm{~km}$ long with $10^{6}$ plates and $L_{B}=17.7 \mathrm{~m}$. Triangles refer to mean DGD values calculated with the FMM. Conversely, circles represent the mean DGD obtained from the RMM. We note the very good agreement with theoretical predictions, which extends the practical validity of (9) also to the RMM, at least in the short-period case.

\section{B. Without the Short-Period Assumption}

We have not been able to find an analytical expression for the mean DGD when the short-period assumption does not hold. In order to investigate the system's behavior in this case, we performed numerical simulations for both the FMM and RMM fiber models, and we calculated the SIRF as defined in (8). We used the sinusoidal spin function reported in Section III-A and estimated the average DGD over an ensemble of 6000 fibers $10 \mathrm{~km}$ long with $10^{6}$ plates, as explained before. We calculated the evolution of the SIRF as a function of spin amplitude for $p=L_{F}=4 \mathrm{~m}$ and for different values of the beat length. Namely, we set $L_{B}=0.9,1.3,1.8,2.7,4.4$, and $17.7 \mathrm{~m}$. We report results for the RMM in Fig. 2 and for the FMM in Fig. 3.

In both figures, the solid curves labeled with letters from a to $\mathrm{f}$ refer to increasing values of the beat length, while the dashed 


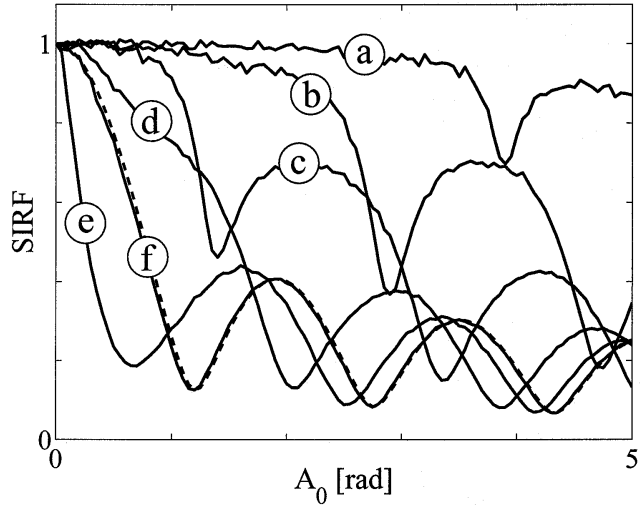

Fig. 3. Numerical estimates for the evolution of the SIRF with the FMM as a function of spin amplitude, for a sinusoidal spin function with $p=L_{F}=4 \mathrm{~m}$. The solid curves, labeled with letters from a to $f$, correspond to $L_{B}=0.9$, $1.3,1.8,2.7,4.4$, and $17.7 \mathrm{~m}$, respectively. The dashed curve is obtained by calculating the SIRF using (11).

curve is obtained calculating $\Phi$ with (11). We find that there is an agreement between the numerical simulations and the theoretical results of the short-period assumption only for the longest value of $L_{B}$. For shorter values of the beat length, the SIRF shows a significant difference, and it is no longer independent of $L_{B}$.

Another remarkable result is the difference between the SIRF curves obtained from the FMM and the RMM. In fact, we see that when the short-period assumption does not hold, the SIRF of the FMM has marked minima, which do not appear when the RMM is used. These differences deserve further investigations, since it seems that the two models of birefringence yield comparable results only in the short-period limit, implying that knowledge of the correct statistical model for fiber birefringence is essential in the design and analysis of spun fibers. For unspun fibers, the mean DGD is independent of the statistical model [12], [20].

\section{DERIVATION OF (9)}

In this section, we describe the derivation of (9) for the FMM fiber model. This derivation, which is somewhat lengthy, is presented in Sections IV-A-D. The point of this derivation is not only to obtain the form of (9). It is also to show that for any value of $p, L_{B}$ and $L_{F}$, and in the reference frame that rotates with the spin rate, the mean polarization dispersion vector of a periodically spun fiber is asymptotically periodic, hence asymptotically stable, when $z \rightarrow \infty$. In order to facilitate the reading of our proof, we summarize the main sections below:

1) Section IV-A. By means of (1) and using the FMM, we derive an equation for the evolution of the mean-square DGD. We find that $\left\langle\Delta \tau^{2}(z)\right\rangle$ depends on $\langle\boldsymbol{\Omega}(z)\rangle$, whose evolution is described by the three-dimensional differential (15).

2) Section IV-B. It is proved that for any periodic spin function and for any initial condition $\langle\boldsymbol{\Omega}(0)\rangle,\langle\boldsymbol{\Omega}(z)\rangle$ tends asymptotically to a periodic orbit with the same periodicity. As a consequence, upon finding such orbit, we determine the behavior of $\left\langle\Delta \tau^{2}(z)\right\rangle$ in the long-length regime.
3) Section IV-C. Owing to the existence of a periodic solution for (15), we may expand this equation in a Fourier series. We then apply the short-period assumption, which allows us approximate (15) with the two-dimensional (2-D) (22).

4) Section IV-D. We solve (22) analytically and find the evolution of $\langle\Delta \tau(z)\rangle$ in the long-length regime.

\section{A. Equation of the Mean-Square DGD}

We start by transforming the reference frame to compensate for both the intrinsic rotation of $\beta(z)$ and the rotation induced by the spin. This transformation is achieved by the matrix $\mathrm{T}(z)=$ $\mathrm{R}_{3}[2 A(z)+\theta(z)]$, where $\mathrm{R}_{3}$ is defined in (7). In the new frame, the dynamical equation reads

$$
\frac{\partial \boldsymbol{\Omega}}{\partial z}=\left(\begin{array}{c}
b \\
0 \\
-2[\alpha(z)+\sigma \eta(z)]
\end{array}\right) \times \boldsymbol{\Omega}+\left(\begin{array}{c}
b_{\omega} \\
0 \\
0
\end{array}\right)
$$

where $b_{\omega}$ is the frequency derivative of $b(\omega)$, and $\alpha(z)$ is the $z$ derivative of $A(z)$. Note that the rotation matrix $\mathrm{T}(z)$ is an orthogonal matrix so that the modulus of $\boldsymbol{\Omega}$ is still equal to the DGD after transformation.

By means of the theory of stochastic differential equations (SDE), we can deduce from (13) an equation for the evolution of the mean-square DGD. Using the Dynkin's formula, we find [21]

$$
\frac{\partial\left\langle\Delta \tau^{2}\right\rangle}{\partial z}=\left\langle\mathcal{A} \Delta \tau^{2}(z)\right\rangle=2 b_{\omega}\left\langle\Omega_{1}(z)\right\rangle
$$

where $\mathcal{A}$, the infinitesimal generator associated with (13), is

$$
\begin{aligned}
\mathcal{A}= & \left(-2 \sigma^{2} \Omega_{1}+2 \alpha \Omega_{2}+b_{\omega}\right) \frac{\partial}{\partial \Omega_{1}} \\
& +\left(-2 \alpha \Omega_{1}-2 \sigma^{2} \Omega_{2}-b \Omega_{3}\right) \frac{\partial}{\partial \Omega_{2}}+b \Omega_{2} \frac{\partial}{\partial \Omega_{3}} \\
& +2 \sigma^{2}\left(\Omega_{2}^{2} \frac{\partial^{2}}{\partial \Omega_{1}^{2}}-2 \Omega_{1} \Omega_{2} \frac{\partial^{2}}{\partial \Omega_{1} \partial \Omega_{2}}+\Omega_{1}^{2} \frac{\partial^{2}}{\Omega_{2}^{2}}\right) .
\end{aligned}
$$

Using Dynkin's formula again, we also find the differential equation

$$
\frac{\partial\langle\boldsymbol{\Omega}\rangle}{\partial z}=\left(\begin{array}{ccc}
-2 \sigma^{2} & 2 \alpha(z) & 0 \\
-2 \alpha(z) & -2 \sigma^{2} & -b \\
0 & b & 0
\end{array}\right)\langle\boldsymbol{\Omega}\rangle+\left(\begin{array}{c}
b_{\omega} \\
0 \\
0
\end{array}\right)
$$

which must be solved in order to determine the evolution of $\left\langle\Delta \tau^{2}(z)\right\rangle$. To accomplish this task, we will use the theory of differential equations with periodic coefficients, which is briefly reviewed in Appendix A. For a thorough treatment of this theory, we refer to [22].

\section{B. Asymptotic Behavior of (15)}

We now prove that (15) has a unique periodic solution with a period of $p$ and that, for any initial condition, the solution of (15) converges asymptotically to the periodic one.

First of all, let us define the quantities

$$
\mathrm{B}(z)=\left(\begin{array}{ccc}
-2 \sigma^{2} & 2 \alpha(z) & 0 \\
-2 \alpha(z) & -2 \sigma^{2} & -b \\
0 & b & 0
\end{array}\right), \quad \boldsymbol{v}=\left(\begin{array}{c}
b_{\omega} \\
0 \\
0
\end{array}\right) .
$$


Recalling that the solution of (15) with generic initial condition $\langle\boldsymbol{\Omega}(0)\rangle$ is given by (A2), and using the Floquet-Lyapunov theorem (A4), we may show that $\langle\boldsymbol{\Omega}(z+p)\rangle$ reads

$$
\begin{aligned}
& \langle\boldsymbol{\Omega}(z+p)\rangle=\langle\boldsymbol{\Omega}(z)\rangle+\mathrm{X}(z) \\
& \left\{[\exp (\mathrm{K} p)-\mathrm{I}]\langle\boldsymbol{\Omega}(0)\rangle+\int_{-p}^{0} \exp (-\mathrm{K} t) \mathrm{F}^{-1}(t) \boldsymbol{v} d t\right\}
\end{aligned}
$$

where $\mathrm{X}(z)=\mathrm{F}(z) \exp (\mathrm{K} z)$ is the fundamental matrix. Notice that the term between curly brackets is bounded and independent of $z$ so that the asymptotic behavior of $\langle\boldsymbol{\Omega}(z+p)\rangle$ is governed by $\mathrm{X}(z)$.

We now prove that $\mathrm{X}(z)$ tends to zero as $z \rightarrow \infty$; consequently, $\langle\boldsymbol{\Omega}(z)\rangle$ tends to be periodic with period $p$, and, as explained in Appendix A, (15) has a unique periodic solution.

To analyze the stability of $X(z)$, we use Lyapunov's second method [22]. First, we define $\alpha_{\max }=\max \{|\alpha(z)|\}$, and we assume that $\alpha_{\max }$ is finite. We now let $\mathrm{B}(z)=\mathrm{C}+\alpha_{\max } \mathrm{Q}(z)$, where

$$
\begin{aligned}
\mathrm{C} & =\left(\begin{array}{ccc}
-2 \sigma^{2} & 0 & 0 \\
0 & -2 \sigma^{2} & -b \\
0 & b & 0
\end{array}\right) \\
\mathrm{Q}(z) & =\left(\begin{array}{ccc}
0 & 2 q(z) & 0 \\
-2 q(z) & 0 & 0 \\
0 & 0 & 0
\end{array}\right)
\end{aligned}
$$

and $\alpha(z)=\alpha_{\max } q(z)$ so that $|q(z)| \leq 1$ for every $z$. The eigenvalues of $\mathrm{C}$ are

$$
\lambda_{1}=-2 \sigma^{2}, \quad \lambda_{2,3}=-2 \sigma^{2} \pm \sqrt{\sigma^{4}-b^{2}}
$$

so that, since $\sigma^{2}>0$ and $b^{2}>0$, the real parts of $\lambda_{i}$ are negative, as required by Lyapunov's second method.

We now define the diagonal matrix $\mathrm{G}_{0}=\operatorname{diag}\left(g_{1}, g_{2}, g_{1}-\right.$ $g_{2}$ ), where $g_{1}$ and $g_{2}$ are arbitrary real numbers that satisfy $g_{1}>$ $g_{2}>0$ so that $\mathrm{G}_{0}$ is positive definite. Let $\mathrm{H}$ be the solution of the equation $\mathrm{HC}+\mathrm{C}^{\top} \mathrm{H}=-\mathrm{G}_{0}$ so that

$$
\mathrm{H}=\left(\begin{array}{ccc}
\frac{g_{1}}{4 \sigma^{2}} & 0 & 0 \\
0 & \frac{g_{1}}{4 \sigma^{2}} & \frac{g_{1}-g_{2}}{2 b} \\
0 & \frac{g_{1}-g_{2}}{2 b} & \frac{g_{1}}{4 \sigma^{2}}+\frac{\left(g_{1}-g_{2}\right) \sigma^{2}}{b^{2}}
\end{array}\right) .
$$

We next define the matrix $\mathrm{G}_{1}(z)=-\mathrm{HQ}(z)-\mathrm{Q}(z)^{\mathrm{T}} \mathrm{H}$. The principal minors of the matrix $\mathrm{G}_{0}+\alpha_{\max } \mathrm{G}_{1}$ are

$$
\begin{aligned}
& \Delta_{1}\left(z, \alpha_{\max }\right)=g_{1}, \quad \Delta_{2}\left(z, \alpha_{\max }\right)=g_{1} g_{2} \\
& \Delta_{3}\left(z, \alpha_{\max }\right)=\frac{g_{2}\left(g_{1}-g_{2}\right)}{b^{2}}\left[b^{2} g_{1}-\alpha_{\max }^{2}\left(g_{1}-g_{2}\right) q^{2}(z)\right] .
\end{aligned}
$$

By Lyapunov's theorem, the trivial solution of (15) is asymptotically stable if $\Delta_{i}\left(z, \alpha_{\max }\right)>0$ for any $z$ and for $i=1,2,3$. Recalling that $g_{1}>g_{2}>0$, we see that indeed $\Delta_{i}\left(z, \alpha_{\max }\right)>0$ for $i=1$ and $i=2$. For $i=3$, we have

$$
\alpha_{\max }^{2}\left(g_{1}-g_{2}\right) q^{2}(z)<b^{2} g_{1} \text {. }
$$

Since $|q(z)| \leq 1$, (17) holds if $\alpha_{\max }^{2}\left(g_{1}-g_{2}\right)<b^{2} g_{1}$, i.e.,

$$
\alpha_{\max }^{2}<b^{2} \frac{g_{1}}{g_{1}-g_{2}} \text {. }
$$

This result states that if $\alpha_{\max }$ is sufficiently small so that the spin function evolves sufficiently slowly, then $\mathrm{X}(z)$ is asymptotically stable, and $\langle\boldsymbol{\Omega}(z)\rangle$ tends to be periodic. Note, however, that, since the number $g_{1} /\left(g_{1}-g_{2}\right)$ can be arbitrarily large, $\mathrm{X}(z)$ is asymptotically stable in any case, and (15) always has a unique periodic solution with a period of $p$.

We conclude that the asymptotic evolution of $\left\langle\Delta \tau^{2}(z)\right\rangle$ is determined by the periodic solution of (15). In fact, let $\left\langle\Omega_{1}(z)\right\rangle$ be the first component of such solution, then owing to (14), the mean-square DGD may be written

$$
\left\langle\Delta \tau^{2}(z)\right\rangle=2 b_{\omega} \int_{0}^{z}\left\langle\Omega_{1}(t)\right\rangle d t \simeq 2 b_{\omega} z \frac{1}{p} \int_{0}^{p}\left\langle\Omega_{1}(t)\right\rangle d t
$$

where we assume, without loss of generality, that $\left\langle\Delta \tau^{2}(0)\right\rangle=$ 0 . The next step is to find the unique periodic solution of (18).

\section{Fourier Expansion of (15)}

Since (15) has a periodic solution with a period of $p$, and since $\mathrm{B}(z)$ is periodic with that same period, we are allowed to expand the terms of (15) in a Fourier series. Therefore, we may write

$$
\begin{aligned}
\left\langle\Omega_{m}(z)\right\rangle & =\sum_{n=-\infty}^{+\infty} \Omega_{m, n} \exp (-j 2 \pi \nu n z), \quad m=1,2,3 \\
\alpha(z) & =\sum_{n=-\infty}^{+\infty} \alpha_{n} \exp (-j 2 \pi \nu n z)
\end{aligned}
$$

where $\nu=1 / p$ is the fundamental frequency. We now insert these expressions in (15), and we equate the harmonics of the same order $k$. The result is

$$
\begin{aligned}
& j 2 \pi \nu k \Omega_{1, k}=2 \sigma^{2} \Omega_{1, k}-\sum_{n=-\infty}^{+\infty} 2 \alpha_{k-n} \Omega_{2, n}-b_{\omega} \delta_{k} \\
& j 2 \pi \nu k \Omega_{2, k}=2 \sigma^{2} \Omega_{2, k}+b \Omega_{3, k}+\sum_{n=-\infty}^{+\infty} 2 \alpha_{k-n} \Omega_{1, n} \\
& j 2 \pi \nu k \Omega_{3, k}=-b \Omega_{2, k}
\end{aligned}
$$

where $\delta_{k}$ is the Kronecker delta. In the case $k=0,(19)$ becomes

$$
\begin{aligned}
2 \sigma^{2} \Omega_{1,0}-\sum_{n=-\infty}^{+\infty} 2 \alpha_{-n} \Omega_{2, n} & =b_{\omega} \\
b \Omega_{3,0}+\sum_{n=-\infty}^{+\infty} 2 \alpha_{-n} \Omega_{1, n} & =0 \\
b \Omega_{2,0} & =0
\end{aligned}
$$

where we have already used the property $\Omega_{2,0}=0$, which is a result of the third equation of (19). As a consequence, the second equation yields

$$
-b \Omega_{3,0}=-b \frac{1}{p} \int_{0}^{p}\left\langle\Omega_{3}(z)\right\rangle d z=\frac{1}{p} \int_{0}^{p} 2 \alpha(z)\left\langle\Omega_{1}(z)\right\rangle d z .
$$

When $k \neq 0$, we can solve for $\Omega_{3, k}$, the third equation of (19), and then insert the result in the second one, finding

$j 2 \pi \nu k\left[1-\left(\frac{b}{2 \pi \nu k}\right)^{2}\right] \Omega_{2, k}=2 \sigma^{2} \Omega_{2, k}$

$$
+\sum_{n=-\infty}^{+\infty} 2 \alpha_{k-n} \Omega_{1, n}
$$


If we assume that $b^{2} \ll(2 \pi \nu)^{2}$, i.e., $p^{2} \ll L_{B}^{2}$, then the previous expression may be simplified as

$$
j 2 \pi \nu k \Omega_{2, k}=2 \sigma^{2} \Omega_{2, k}+\sum_{n=-\infty}^{+\infty} 2 \alpha_{k-n} \Omega_{1, n} .
$$

This result, combined with (20), implies

$$
j 2 \pi \nu k \Omega_{2, k}=2 \sigma^{2} \Omega_{2, k}+\sum_{n=-\infty}^{+\infty} 2 \alpha_{k-n} \Omega_{1, n}+b \Omega_{3,0} \delta_{k, 0} .
$$

We see that this equation, together with the first one of (19), form a new system, completely decoupled from the third equation of (19). This 2-D system corresponds to the differential equation

$$
\frac{d \boldsymbol{y}}{d z}=\left(\begin{array}{cc}
-2 \sigma^{2} & 2 \alpha(z) \\
-2 \alpha(z) & -2 \sigma^{2}
\end{array}\right) \boldsymbol{y}(z)+\left(\begin{array}{c}
b_{\omega} \\
-b \Omega_{3,0}
\end{array}\right)
$$

where $\boldsymbol{y}(z)=\left(\left\langle\Omega_{1}(z)\right\rangle,\left\langle\Omega_{2}(z)\right\rangle\right)^{\top}$. Upon solving (22), we find the evolution of $\left\langle\Omega_{1}(z)\right\rangle$ and, hence, the evolution of $\left\langle\Delta \tau^{2}(z)\right\rangle$.

\section{Solution of the 2-D System (22)}

In order to solve (22), we start by considering its fundamental matrix $\mathrm{Y}(z)$, which obeys the equation

$$
\frac{d \mathrm{Y}}{d z}=\left(\begin{array}{cc}
-2 \sigma^{2} & 2 \alpha(z) \\
-2 \alpha(z) & -2 \sigma^{2}
\end{array}\right) \mathrm{Y}(z)
$$

with the initial condition $Y(0)=I$ (the identity matrix). As a consequence, the explicit solution of $Y(z)$ reads as

$$
\mathrm{Y}(z)=\exp \left(-2 \sigma^{2} z\right)\left(\begin{array}{cc}
\cos 2 A(z) & \sin 2 A(z) \\
-\sin 2 A(z) & \cos 2 A(z)
\end{array}\right) .
$$

Note that $Y(z)$ is asymptotically stable as long as $\sigma \neq 0$ so that (22) has a unique periodic solution with a period of $p$, which may be written as (see Appendix A)

$$
\boldsymbol{y}(z)=[\mathrm{I}-\mathrm{R}(z, 0)]^{-1} \int_{0}^{p} \mathrm{R}(z, p-t) \boldsymbol{f} d t
$$

where $\boldsymbol{f}=\left(b_{\omega},-b \Omega_{3,0}\right)^{\mathrm{T}}$ and $\mathrm{R}(z, t)=\mathrm{Y}(z+p) \mathrm{Y}^{-1}(z+t)$. Explicitly, we now find

$$
\begin{aligned}
& \left\langle\Omega_{1}(z)\right\rangle=\frac{\gamma b_{\omega}}{2 \sigma^{2} p} \int_{0}^{p} \exp \left(-2 \sigma^{2} t\right) \cos [2 A(z)-2 A(z-t)] d t \\
& -\frac{\gamma b}{2 \sigma^{2} p} \Omega_{3,0} \int_{0}^{p} \exp \left(-2 \sigma^{2} t\right) \sin [2 A(z)-2 A(z-t)] d t
\end{aligned}
$$

with $\gamma=2 \sigma^{2} p /\left[1-\exp \left(-2 \sigma^{2} p\right)\right]$.

Equation (23) is not self-consistent, because $\Omega_{3,0}$ is still unknown. However, this obstacle may be overcome by inserting (23) into (21) and solving for $-b \Omega_{3,0}$, to obtain

$$
-b \Omega_{3,0}=b_{\omega} \frac{\mathcal{C}_{\alpha}}{2 \sigma^{2}-\mathcal{S}_{\alpha}}
$$

where

$$
\begin{aligned}
\mathcal{C}_{\alpha}= & \frac{\gamma}{p^{2}} \int_{0}^{p} \int_{0}^{p} 2 \alpha(t) \exp \left(-2 \sigma^{2} u\right) \\
& \cos [2 A(t)-2 A(t-u)] d u d t \\
\mathcal{S}_{\alpha}= & \frac{\gamma}{p^{2}} \int_{0}^{p} \int_{0}^{p} 2 \alpha(t) \exp \left(-2 \sigma^{2} u\right) \\
& \times \sin [2 A(t)-2 A(t-u)] d u d t .
\end{aligned}
$$

In this way, we have completely determined the first component of the periodic solution of (15).

From (18), it follows that we must determine the average over a period of $\left\langle\Omega_{1}(z)\right\rangle$, which can be calculated from (23) and (24), to yield

$$
\frac{1}{p} \int_{0}^{p}\left\langle\Omega_{1}(t)\right\rangle d t=\frac{b_{\omega}}{2 \sigma^{2}} \frac{2 \sigma^{2} \mathcal{C}-\mathcal{C S}_{\alpha}+\mathcal{S C}_{\alpha}}{2 \sigma^{2}-\mathcal{S}_{\alpha}}
$$

with $\mathcal{C}$ and $\mathcal{S}$ being defined as in (10). By means of some algebra that we defer to Appendix B, we find that

$$
\mathcal{C}_{\alpha}=2 \sigma^{2} \mathcal{S}, \quad \mathcal{S}_{\alpha}=2 \sigma^{2}(1-\mathcal{C})
$$

so that the average over a period of $\left\langle\Omega_{1}(z)\right\rangle$ is

$$
\frac{1}{p} \int_{0}^{p}\left\langle\Omega_{1}(t)\right\rangle d t=\frac{b_{\omega}}{2 \sigma^{2}} \frac{\mathcal{C}^{2}+\mathcal{S}^{2}}{\mathcal{C}} .
$$

Then, owing to (18), we obtain

$$
\left\langle\Delta \tau^{2}(z)\right\rangle=\frac{b_{\omega}^{2}}{\sigma^{2}} \frac{\mathcal{C}^{2}+\mathcal{S}^{2}}{\mathcal{C}} z .
$$

Finally, recalling that the DGD is a Maxwellian random variable, we arrive at (9).

\section{CONCLUSION}

In this paper, we have derived an analytical formula for the mean DGD of randomly birefringent spun fibers, in the case of periodic spin functions with period $p$ that obey the short-period assumption, $p^{2} \ll L_{B}^{2}$. The analysis has been carried out in the framework of the fixed-modulus model of birefringence, which assumes that only the birefringence orientation varies randomly, while its modulus is fixed. However, for completeness, the results provided by the FMM have been numerically compared with those given by the random-modulus model of birefringence.

It turns out that the two models yield the same result for the DGD, as long as the short-period assumption is satisfied. On the contrary, when the short-period assumption is not satisfied, there is a remarkable difference in the results that are yielded by the two models. This difference implies that, in order to analyze in more detail the effects of the spin process, we must know which model of birefringence best describes a fiber.

When the short-period assumption holds,(9) shows that there exist spin amplitudes such that the DGD reaches a local minimum. This result is indeed useful for optimized design of low-PMD fibers. Moreover, (9) is a first step in a more comprehensive analysis of transmission through spun fibers. 


\section{APPENDIX A}

\section{LINEAR DIFFERENTIAL EQUATIONS WITH PERIODIC COEFFICIENTS}

Consider a first-order linear differential equation

$$
\frac{d \boldsymbol{y}}{d t}=\mathrm{A}(t) \boldsymbol{y}(t)+\boldsymbol{f}(t)
$$

where $\boldsymbol{y}(t)$ and $\boldsymbol{f}(t)$ are $n$-dimensional vector functions, and $\mathrm{A}(t)$ is a $n \times n$ matrix function. The solution of (A1) with initial condition $\boldsymbol{y}(0)$ is [22]

$$
\boldsymbol{y}(t)=\mathrm{Y}(t)\left[\boldsymbol{y}(0)+\int_{0}^{t} \mathrm{Y}^{-1}(\tau) \boldsymbol{f}(\tau) d \tau\right]
$$

where $\mathrm{Y}(t)$ is the fundamental matrix associated with (A1), which is an $n \times n$ matrix function obeying the homogeneous equation

$$
\frac{d \mathrm{Y}}{d t}=\mathrm{A}(t) \mathrm{Y}(t)
$$

with initial condition $Y(0)=I$ (the identity matrix).

When both $\mathrm{A}(t)$ and $\boldsymbol{f}(t)$ are periodic with period $p$, (A1) belongs to the class of Hill equations that are linear differential equations with periodic coefficients [22]. We must determine whether there are conditions such that the corresponding solutions of (A1) are periodic with the same period $p$ by examining the properties of the fundamental matrix.

By the Floquet-Lyapunov theorem [22], the fundamental matrix of a system of differential equations with periodic coefficients may be expressed as

$$
\mathrm{Y}(t)=\mathrm{F}(t) \exp (\mathrm{K} t)
$$

where $F(t)$ is a periodic nonsingular matrix function with period $p$, such that $\mathrm{F}(0)=\mathrm{I}$ and $\mathrm{K}$ is a constant matrix. The eigenvalues of $\mathrm{K}$ are known as the characteristic exponents of (A1).

For what concerns our problem, we consider only the case where all the characteristic exponents have negative real parts. Then, it may be shown that (A1) has a unique periodic solution, corresponding to a definite initial condition [22]. Consequently, such solution can be written as

$$
\boldsymbol{y}(t)=[\mathrm{I}-\mathrm{R}(t, 0)]^{-1} \int_{0}^{p} \mathrm{R}(t, p-\tau) \boldsymbol{f}(t-\tau) d \tau
$$

where $\mathrm{R}(t, s)=\mathrm{Y}(t+p) \mathrm{Y}^{-1}(t+s)$. Note that in (A5), the initial condition does not appear explicitly because the solution is unique

We remark also that when all the characteristic exponents have negative real parts, then $Y(t)$ is asymptotically stable, meaning that it converges to zero as $t \rightarrow \infty$. Conversely, if $Y(t)$ is asymptotically stable, then all the characteristic exponents have negative real parts, and (A1) has a unique periodic solution with period $p$.

\section{APPENDIX B}

\section{Calculus of $\mathcal{C}_{\alpha}$ and $\mathcal{S}_{\alpha}$}

In this appendix, we prove (25). We only explicitly calculate $\mathcal{C}_{\alpha}$, because one can calculate $\mathcal{S}_{\alpha}$ in exactly the same way.
As a first step, we derive a relationship for $\mathcal{S}$ that will be used later. Let us perform the change of variable $x=t$ and $y=t-u$, so that (10) reads

$$
\mathcal{S}=\int_{0}^{p} \int_{x-p}^{x} g(x, y) d y d x
$$

where we set

$$
g(x, y)=\frac{\gamma}{p^{2}} \exp \left[-2 \sigma^{2}(x-y)\right] \sin [2 A(x)-2 A(y)] .
$$

Let us define the bidimensional areas

$$
\begin{aligned}
& \mathbb{T}_{1}=\{(x, y): 0 \leq x \leq p, 0 \leq y \leq x\} \\
& \mathbb{T}_{2}=\{(x, y): 0 \leq x \leq p, x-p \leq y \leq 0\} \\
& \mathbb{T}_{3}=\{(x, y): 0 \leq x \leq p, x \leq y \leq p\} .
\end{aligned}
$$

Note that $\mathbb{T}_{2}=\mathbb{T}_{3}-(p, 0)$ so that (B6) can be rearranged as follows, recalling that the spin function has a period of $p$ :

$$
\mathcal{S}=\int_{\mathbb{T}_{1}} g(x, y) d y d x+\exp \left(-2 \sigma^{2} p\right) \int_{\mathbb{T}_{3}} g(x, y) d y d x .
$$

Consider now $\mathcal{C}_{\alpha}$; by making the change of variables $(t, u) \rightarrow$ $(x, y)$ above, and using the definition of $\mathbb{T}_{i}$, we may write

$$
\mathcal{C}_{\alpha}=\int_{\mathbb{T}_{1}} f(x, y) d y d x+\exp \left(-2 \sigma^{2} p\right) \int_{\mathbb{T}_{3}} f(x, y) d y d x
$$

where

$$
f(x, y)=\frac{\gamma}{p^{2}} 2 \alpha(x) \exp \left[-2 \sigma^{2}(x, y)\right] \cos [2 A(x)-2 A(y)] .
$$

To solve the first integral of (B11), we start by changing the order of integration, finding

$$
\begin{aligned}
\int_{\mathbb{T}_{1}} f(x, y) d y d x=\frac{\gamma}{p^{2}} \int_{0}^{p} \exp \left(2 \sigma^{2} y\right) \int_{y}^{p} 2 \alpha(x) \\
\exp \left(-2 \sigma^{2} x\right) \cos [A(x)-A(y)] d x d y .
\end{aligned}
$$

Then, the innermost integral may be evaluated by parts, yielding

$$
\begin{aligned}
\int_{\mathbb{T}_{1}} f(x, y) d y d x=2 \sigma^{2} \int_{\mathbb{T}_{1}} g(x, y) d x d y+\frac{\gamma}{p^{2}} \exp \left(-2 \sigma^{2} p\right) \\
\int_{0}^{p} \exp \left(2 \sigma^{2} y\right) \sin [2 A(p)-2 A(y)] d y .
\end{aligned}
$$

The second integral of (B11) can be evaluated with the same procedure, and the result is

$$
\begin{array}{r}
\int_{\mathbb{T}_{3}} f(x, y) d y d x=2 \sigma^{2} \int_{\mathbb{T}_{3}} g(x, y) d x d y-\frac{\gamma}{p^{2}} \int_{0}^{p} \exp \left(2 \sigma^{2} y\right) \\
\sin [2 A(0)-2 A(y)] d y .
\end{array}
$$

Finally, recalling the $A(0)=A(p)$, we obtain

$$
\mathcal{C}_{\alpha}=2 \sigma^{2} \int_{\mathbb{T}_{1}} g(x, y) d y d x
$$

$$
+2 \sigma^{2} \exp \left(-2 \sigma^{2} p\right) \int_{\mathbb{T}_{3}} g(x, y) d y d x
$$


so that, upon comparing this expression with (B10), the property $\mathcal{C}_{\alpha}=2 \sigma^{2} \mathcal{S}$ is proved.

\section{APPENDIX C \\ SIMPLIFICATION OF (10)}

In some cases, the integrals (10) can be simplified.

The first case that we consider is when $L_{F} \gg p$ so that $\exp \left(-2 \sigma^{2} u\right) \simeq 1$ for all $u \in[0, p]$, and $\gamma \simeq 1$; this case was considered also by Chen et al. [17] using secular perturbation theory. Then, $\mathcal{S}$ reads as

$$
\mathcal{S}=\frac{1}{p^{2}} \int_{0}^{p} \int_{0}^{p} \sin [2 A(t)-2 A(t-u)] d t d u .
$$

By introducing the new variables $x=t$ and $y=t-u$, this integral can be rearranged as

$$
\begin{aligned}
\mathcal{S} & =\frac{1}{p^{2}} \int_{0}^{p} \int_{x-p}^{x} \sin [2 A(x)-2 A(y)] d y d x \\
& =\frac{1}{p^{2}} \int_{0}^{p} \int_{0}^{p} \sin [2 A(x)-2 A(y)] d y d x
\end{aligned}
$$

where the last equality holds because $s(y)=\sin [2 A(x)-$ $2 A(y)]$ is a periodic function with a period of $p$. Finally, using the properties of trigonometric functions, we obtain

$$
\begin{aligned}
\mathcal{S}=\frac{1}{p^{2}}\left\{\int_{0}^{p} \sin 2 A(x) d x \int_{0}^{p} \cos 2 A(y) d y\right. & \left.\int_{0}^{p} \cos 2 A(x) d x \int_{0}^{p} \sin 2 A(y) d y\right\}=0 .
\end{aligned}
$$

Under the same hypothesis and using the same technique, we find

$$
\mathcal{C}=\left[\frac{1}{p} \int_{0}^{p} \cos 2 A(u) d u\right]^{2}+\left[\frac{1}{p} \int_{0}^{p} \sin 2 A(u) d u\right]^{2} .
$$

We now consider the case of periodic spin functions with only odd harmonics. This class of functions satisfies the symmetry $A(z)=-A(z+p / 2)$. It is easy to see that also the function $s(t, u)=\sin [2 A(t)-2 A(t-u)]$ is characterized by the same symmetry, i.e., $s(t, u)=-s(-t+p / 2, u)$. Using $s(t, u)$, the double integral, $\mathcal{S}$ in (10) can be rearranged as

$$
\mathcal{S}=\frac{\gamma}{p^{2}} \int_{0}^{p} \exp \left(-2 \sigma^{2} u\right)
$$

$$
\left[\int_{0}^{p / 2} s(t, u) d t+\int_{p / 2}^{p} s(t, u) d t\right] d u
$$

then, in the second of the inner integrals, we introduce the new variable $\tau=t-p / 2$, obtaining

$$
\int_{p / 2}^{p} s(t, u) d t=\int_{0}^{p / 2} s\left(\tau+\frac{p}{2}, u\right) d \tau=-\int_{0}^{p / 2} s(\tau, u) d \tau
$$

and consequently, $\mathcal{S}=0$.

Finally, we consider the spin functions that satisfy the symmetry $A(z+q)=A(-z+q)$ and show that this property yields $\mathcal{S}=0$. In the following, we report the proof for the case $q=0$, but it can be extended in a straightforward way to the case $q \neq 0$ which differs only for a translation. By introducing again the variables $x=t$ and $y=t-u$, the quantity $\mathcal{S}$ reads as in (B6) and can be rearranged as reported in (B10), with $\mathbb{T}_{1}$ and $\mathbb{T}_{3}$ defined in (B9). We note that the two bidimensional areas $\mathbb{T}_{1}$ and $\mathbb{T}_{3}$ are both symmetric with respect to the straight line $y=-x+p$. As a consequence, both the integrals in (B10) are zero if the function $g(x, y)$ presents an odd symmetry with respect to that straight line, as is the case for the spin functions that belong to the particular class we are considering. In fact, any point $(x, y)$ on the plane is the symmetric dual of the point $\left(x^{\prime}, y^{\prime}\right)=(p-y, p-x)$ with respect to the straight line $y=-x+p$. Then, the function $g\left(x^{\prime}, y^{\prime}\right)$ can be rearranged as

$$
\begin{aligned}
g\left(x^{\prime}, y^{\prime}\right) & =g(p-y, p-x) \\
& =\frac{\gamma}{p^{2}} \exp \left[-2 \sigma^{2}(p-y-p+x)\right] s(p-y, p-x) \\
& =\frac{\gamma}{p^{2}} \exp \left[-2 \sigma^{2}(x-y)\right] s(-y,-x)=-g(x, y)
\end{aligned}
$$

where $s(x, y)=\sin [2 A(x)-2 A(y)]$ and the last equality holds because, in the case we are considering, $s(-y,-x)=s(x, y)$. As a consequence, $\mathcal{S}=0$ and $\Phi=\sqrt{\mathcal{C}}$.

\section{ACKNOWLEDGMENT}

The research was carried out in the framework of the agreement between Università di Padova and Istituto Superiore delle Comunicazioni e Tecnologie dell'Informazione. The authors would like to acknowledge the Laboratory for Physical Sciences (College Park, MD), the Laboratory for Telecommunications Sciences (Adelphi, MD), as well as the Department of Education and the National Science Foundation.

\section{REFERENCES}

[1] S. C. Rashleigh and R. Ulrich, "Polarization mode dispersion in single-mode fibers," Opt. Lett., vol. 2, pp. 60-62, 1978.

[2] C. D. Poole and J. Nagel, "Polarization effects in lightwave systems," in Optical Fiber Telecommunications, I. P. Kaminow and T. Koch, Eds. San Diego, CA: Academic, 1997.

[3] M. A. Santoro and C. D. Poole, "Polarization scrambling using a short piece of high-birefringence optical fiber and a multifrequency laser diode," J. Lightwave Technol., vol. 12, pp. 288-293, Feb. 1994.

[4] H. Sunnerud, C. Xie, M. Karlsson, R. Samuelsson, and P. A. Andrekson, "A comparison between different PMD compensation techniques," $J$. Lightwave Technol., vol. 20, pp. 368-378, Mar. 2002.

[5] A. J. Barlow, J. J. Ramskov-Hansen, and D. N. Payne, "Birefringence and polarization mode-dispersion in spun single-mode fibers," Appl. Opt., vol. 20, pp. 2962-2968, 1981.

[6] A. Hart, R. G. Huff, and K. L. Walker, "Method of making a fiber having low polarization mode dispersion due to a permanent spin," U.S. Pat. $5298047,1994$.

[7] — - "Article comprising optical fiber having low polarization mode dispersion, due to permanent spin," U.S. Pat. 5418 881, 1995.

[8] M. J. Li and D. A. Nolan, "Fiber spin-profile designs for producing fibers with low polarization mode dispersion," Opt. Lett, vol. 23, pp. 1659-1661, 1998.

[9] A. Galtarossa, L. Palmieri, and A. Pizzinat, "Optimized spinning design for low PMD fibers: An analytical approach," J. Lightwave Technol., vol. 19, pp. 1502-1512, Oct. 2001. 
[10] A. Galtarossa, P. Griggio, A. Pizzinat, and L. Palmieri, "Calculation of mean differential group delay of periodically spun randomly birefringent fibers," Opt. Lett., vol. 27, pp. 692-694, 2002.

[11] C. D. Poole, J. H. Winters, and J. A. Nagel, "Dynamical equation for polarization dispersion," Opt. Lett., vol. 6, pp. 372-374, 1991.

[12] P. K. A. Wai and C. R. Menyuk, "Polarization mode dispersion, decorrelation, and diffusion in optical fibers with randomly varying birefringence," J. Lightwave Technol., vol. 14, pp. 148-157, Feb. 1996.

[13] A. Galtarossa and L. Palmieri, "Measure of twist-induced circular birefringence in long single-mode fibers: Theory and experiments," J. Lightwave Technol., vol. 20, pp. 1149-1159, July 2002.

[14] A. Galtarossa, L. Palmieri, M. Schiano, and T. Tambosso, "Statistical characterization of fiber random birefringence," OSA Opt. Lett., vol. 25 , pp. 1322-1324, 2000 .

[15] — " "Measurement of birefringence correlation length in long single-mode fibers," OSA Opt. Lett., vol. 26, pp. 962-964, 2001.

[16] W. H. Press, S. A. Teukolsky, W. T. Vetterling, and B. P. Flannery, Numerical Recipes in C: The Art of Scientific Computing. Cambridge: Cambridge Univ. Press, 1992.

[17] X. Chen, M.-J. Li, and D. A. Nolan, "Polarization mode dispersion of spun fibers: An analytical solution," Opt. Lett., vol. 27, pp. 294-296, 2002.

[18] A. Galtarossa, L. Palmieri, and A. Pizzinat, "Low-PMD spun fibers," in Proc. Venice Summer School on Polarization Mode Dispersion, Venice, Italy, 2002, Paper Th-08.

[19] F. Corsi, A. Galtarossa, and L. Palmieri, "Analytical treatment of polarization dispersion in single-mode fibers by means of backscattering signal," J. Opt. Soc. Amer. A, vol. 16, pp. 574-583, 1999.

[20] C. R. Menyuk and P. K. A. Wai, "Polarization evolution and dispersion in fibers with spatially varying birefringence," J. Opt. Soc. Amer. B, vol. 11, pp. 1288-1296, 1994.

[21] B. Øksendal, Stochastic Differential Equations. Berlin, Germany: Springer-Verlag, 2000.

[22] V. A. Yakubovich and V. M. Starzhinskii, Linear Differential Equations With Periodic Coefficients. New York: Wiley, 1975.

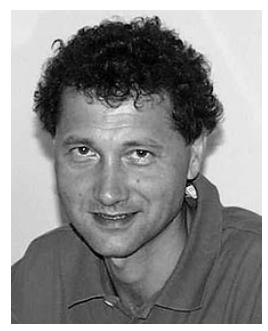

Andrea Galtarossa (M'88) received the degree in electronic engineering from the University of Padova, Italy, in 1984.

In 1986, he received a Postgraduate Fellowship from Telettra Spa, Vimercate (IT), Italy, for research in WDM components. In 1987, he joined Saifo srl, Padova, a private company involved in measurements and teaching in optical systems. In 1990, he became an Assistant Professor in Electromagnetic Fields at the Department of Information Engineering, University of Padova. In 1998, he became an Associate Professor in Microwave at the same university. He is the author or coauthor of more than 60 papers and his current research activity is mainly in birefringent fibers and PMD measurements and modeling.



Luca Palmieri was born in Belluno, Italy, in 1971. $\mathrm{He}$ received the degree in electronic engineering and the Ph.D. degree from the University of Padova, Italy, in 1996 and 2000, respectively.

From 2000 to 2002, he has been a Research Fellow at the University of Udine, Italy, and presently he is Research Fellow at the University of Padova. His research activity is mainly focused on polarization mode dispersion (PMD) causes and effects, and spans from birefringence and PMD measurements techniques to PMD compensation and design of

low-PMD optical fibers.

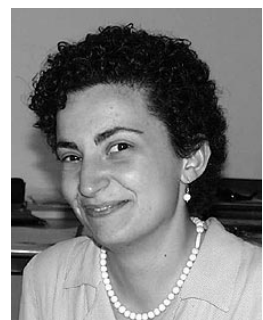

Anna Pizzinat was born in Treviso, Italy, in 1974 She received the Master degree in electronic engineering and the Ph.D. degree from the University of Padova, Italy, in 1999 and 2003, respectively.

In 2002, she spent six months at the University of Maryland Baltimore County as a Visiting Student. In April 2003, she received the award as Best Young Researcher in the Department of Information Engineering, Padova, where she currently works. Her research interests include the study of fibers with low polarization mode dispersion, and the analysis and design of high-bit-rate optical transmission systems.

Brian S. Marks, photograph and biography not available at the time of publication.

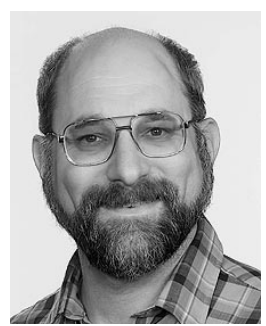

Curtis R. Menyuk (F'98) as born on March 26, 1954. He received the B.S. and M.S. degrees from the Massachusetts Institute of Technology (MIT), Cambridge, in 1976 and the Ph.D. degree from the University of California at Los Angeles (UCLA) in 1981.

He has worked as a Research Associate at the University of Maryland, College Park, and at Science Applications International Corporation, McLean, VA. In 1986, he became an Associate Professor in the Department of Electrical Engineering at the University of Maryland Baltimore County (UMBC), and he was the founding member of this department. In 1993, he was promoted to Professor. He was on partial leave from UMBC from fall 1996 until fall 2002. From 1996 to 2001, he worked part-time for the Department of Defense, codirecting the Optical Networking program at the DoD Laboratory for Telecommunications Sciences, Adelphi, MD, from 1999 to 2001. From 2001 to 2002, he was Chief Scientist at PhotonEx Corporation. For the last 15 years, his primary research area has been theoretical and computational studies of fiber optic communications. He has authored or coauthored more than 160 archival journal publications as well as numerous other publications and presentations. He has also edited two books. The equations and algorithms that he and his research group at UMBC have developed to model optical fiber transmission systems are used extensively in the telecommunications industry.

Dr. Menyuk is a Member of the Society for Industrial and Applied Mathematics and the American Physical Society. He is a Fellow of the Optical Society of America (OSA) and is a former UMBC Presidential Research Professor. 